\title{
Economic Burden
}

National Cancer Institute

\section{Source}

National Cancer Institute. Economic Burden. NCI Thesaurus. Code C19250.

Refers to the costs required to deal with a specific situation (e.g., disease burden). 